\title{
The spin state of iron in minerals of Earth's lower mantle
}

\author{
Wolfgang Sturhahn \\ Advanced Photon Source, Argonne National Laboratory, Lemont, Illinois, USA
}

Jennifer M. Jackson

Department of Geology, University of Illinois at Urbana-Champaign, Urbana, Illinois, USA

Jung-Fu Lin

Geophysical Laboratory, Carnegie Institution of Washington, Washington, D. C., USA

Received 23 February 2005; revised 10 April 2005; accepted 23 May 2005; published 23 June 2005.

[1] The spin state of Fe(II) and Fe(III) at temperatures and pressures typical for the Earth's lower mantle is discussed. We predict an extended high-spin to low-spin crossover region along the geotherm for Fe-dilute systems depending on crystal-field splitting, pairing energy, and cooperative interactions. In particular, spin transitions in ferromagnesium silicate perovskite and ferropericlase, the dominant lower mantle components, should occur in a wide temperaturepressure range. We also derive a gradual volume change associated with such transitions in the lower mantle. The gradual density changes and the wide spin crossover regions seem incompatible with lower mantle stratification resulting from a spin transition. Citation: Sturhahn, W., J. M. Jackson, and J.-F. Lin (2005), The spin state of iron in minerals of Earth's lower mantle, Geophys. Res. Lett., 32, L12307, doi:10.1029/ 2005 GL022802.

\section{Introduction}

[2] The chemical composition, the elastic and transport properties, and the thermodynamic parameters of Earth's deep interior are of general importance to geochemical modeling, geodynamic simulation, and interpretation of seismic wave observations [Kellogg et al., 1999; van der Hilst and Kárason, 1999; Trampert et al., 2004]. Most of the minerals and polymorphs expected in Earth's mantle are believed to incorporate low concentrations of $\mathrm{Fe}(\mathrm{II})$ and/or Fe(III) of about 10 atomic $\%$ or less. The relevance of the Fe spin states in the major lower mantle constituents, ferropericlase $\mathrm{Mg}_{1-x} \mathrm{Fe}_{x} \mathrm{O}$ with $0.1 \leq x \leq 0.25$ (hereafter referred to as $\mathrm{Fp}$ ) and ferromagnesium silicate perovskite $\mathrm{Mg}_{1-y} \mathrm{Fe}_{y} \mathrm{SiO}_{3}$ with $0.05 \leq y \leq 0.15$ (hereafter referred to as Pv) [Liu, 1975; McCammon et al., 1998], with respect to density, iron partitioning, partial melting, radiative thermal conductivity, and compositional layering in the lower mantle has been emphasized previously [Shannon and Prewitt, 1969; Gaffney and Anderson, 1973; Sherman, 1988, 1991; Sherman and Jansen, 1995; Badro et al., 2003, 2004; Li et al., 2004]. Experimental studies using $\mathrm{x}$-ray emission spectroscopy observed an electronic configuration change of the Fe in Fp with $x=0.17$ between 60 and $70 \mathrm{GPa}$ [Badro et al., 2003]. In Pv with $y=0.1$, two changes of the spin state of the iron at $70 \mathrm{GPa}$ and $120 \mathrm{GPa}$

Copyright 2005 by the American Geophysical Union. 0094-8276/05/2005GL022802\$05.00 were interpreted as partial and full spin transitions of $\mathrm{Fe}(\mathrm{II})$, respectively [Badro et al., 2004]. Using the same experimental technique on $\mathrm{Pv}$ of similar composition, as well as on an Al-bearing $\mathrm{Pv}$, an independent study reported a gradual transformation of the iron from an initial high-spin state toward a lower spin state [Li et al., 2004]. A study using synchrotron Mössbauer spectroscopy on Pv with $y=$ 0.05 and $y=0.1$ could not detect a change in the spin state of $\mathrm{Fe}$ (II) up to $120 \mathrm{GPa}$, however, indications for a changing spin state of $\mathrm{Fe}(\mathrm{III})$ around $70 \mathrm{GPa}$ were reported [Jackson et al., 2005]. All these experiments were performed at room temperature. Density-functional calculations of the electronic ground state of $\left(\mathrm{FeO}_{6}\right)^{10-}$ and $\left(\mathrm{FeMg}_{12} \mathrm{O}_{14}\right)^{2-}$ clusters suggested that the iron in Fp undergoes a pressure-dependent transition from a highspin to a low-spin configuration [Sherman, 1988, 1991; Sherman and Jansen, 1995]. Density-functional calculations on wüstite [Cohen et al., 1997] suggested that iron in $\mathrm{Pv}$ should remain in the high-spin state up to much higher pressures than found in the lower mantle. In both cases, the existence or absence of spin transitions was concluded from the pressure dependence of the electronic ground states. The temperature dependence of the transition pressure was extrapolated into the lower mantle using a Clausius-Clapeyron relation under the assumption that the transition persists at high temperatures [Sherman, 1988; Badro et al., 2004; Li et al., 2004]. Clearly, theoretical and experimental studies have provided valuable and often detailed information on the effect of pressure on the spin state of $\mathrm{Fe}$ in oxides and silicates, but the effect of temperature on the population of the spin states has not been considered.

[3] In this paper, we explore the temperature effect on spin states for minerals in Earth's lower mantle like Fp and Pv: the change from a high-spin to a low-spin state (or vice versa) can occur as a continuous crossover rather than a sudden transition. We will use crystal field theory and an extended Bragg-Williams mean-field theory to model the effects of the host mineral and of the iron-iron interactions on the $3 \mathrm{~d}$ energy levels of the iron. If the Fe concentration of the mineral is of the order of 10 atomic $\%$ or less, the average iron-iron distance becomes large enough to result in negligible spin-spin interactions between neighboring iron atoms, and a continuous crossover or "spin equilibrium" is expected [Gütlich and Goodwin, 2004]. The application of these concepts allows us to express the influence of temperature and discuss the implications for a potential strati- 

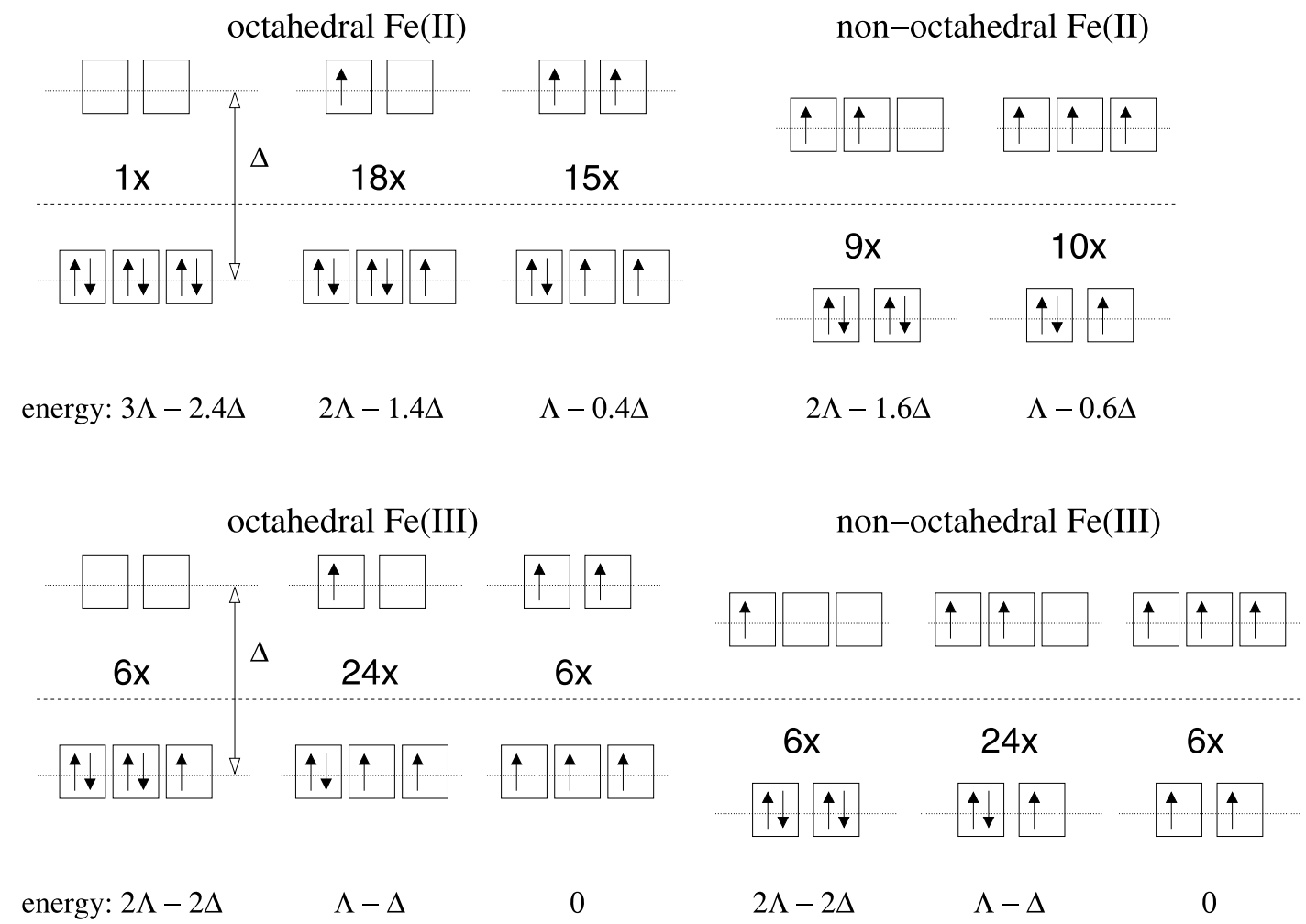

Figure 1. Configurations of the $3 \mathrm{~d}$ electrons of $\mathrm{Fe}(\mathrm{II})$ and $\mathrm{Fe}(\mathrm{III})$, their degeneracies, and energies. The lowest energy configurations for octahedral and other symmetries are shown. The dashed lines represent the energy of the $3 \mathrm{~d}$ electrons in a spherical symmetry.

fication of the lower mantle caused by the spin state of iron in minerals.

\section{Interacting Spin Ensemble}

[4] Isolated $\mathrm{Fe}(\mathrm{II})$ and $\mathrm{Fe}(\mathrm{III})$ in a host lattice experience a change in the electronic configuration of the $3 \mathrm{~d}$ shell that is characteristic to the local environment [Burns, 1993]. The lowest energy configurations for the $3 \mathrm{~d}$ electrons of $\mathrm{Fe}(\mathrm{II})$ and $\mathrm{Fe}(\mathrm{III})$ in nondistorted octahedral, tetrahedral, cubic, and dodecahedral environments are shown in Figure 1. The assumption of perfect symmetries allows us to describe the energy levels with only one parameter, $\omega=\Delta-\Lambda$, the difference between the crystal-field splitting energy $\Delta>0$ and the exchange energy $\Lambda>0$ (also called the spin-pairing energy) which is mostly independent of the local environment. For reduced symmetries, the energy degeneracy is partially or fully lifted, and a larger number of parameters is required. Values for $\Delta$ and $\Lambda$ are typically 1 to $2 \mathrm{eV}$ [Burns, 1993], and for temperatures of the mantle $\beta \Delta \gg 1$ and $\beta \Lambda \gg 1$ with $\beta=1 /\left(k_{B} T\right)$ as the inverse temperature. However the energy differences of the configurations in Figure 1 are multiples of $\omega$, and each configuration may occur with reasonable probability in a thermally equilibrated material. If the interaction between the iron atoms is negligible, the influence of the host lattice is well described by values of $\omega$. Such an assumption is justified for very dilute systems but will probably fail in iron-rich compounds like $\mathrm{FeS}$ or $\mathrm{FeO}$. The minerals that are relevant to the lower mantle are not iron rich, but the influence of weak interactions may still be noticeable. We will include interaction effects in a simple mean-field model based on the Bragg-Williams theory that was originally developed to describe orderdisorder transitions in alloys [Williams, 1935].

[5] Assume that a total number of $N$ iron atoms in the crystal can occupy energetically different spin states described by an index $i$ and that the fraction of iron atoms in such a state is given by $\eta_{i}$ with $\sum_{i} \eta_{i}=1$. A combinatorial analysis and the use of Stirling's formula provides for the entropy of the ensemble

$$
S=-k_{B} N \sum_{i} \eta_{i} \ln \frac{\eta_{i}}{g_{i}}
$$

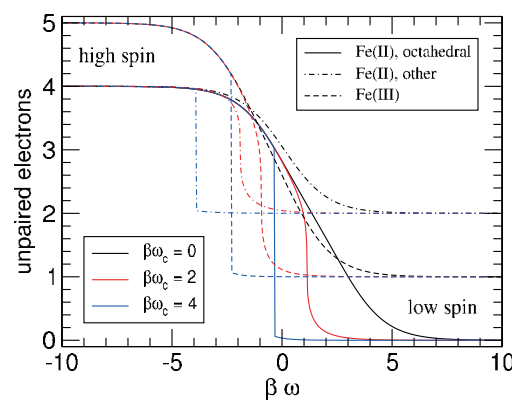

Figure 2. The average number of unpaired electrons for increasing values of the reduced coupling parameter $\beta \omega_{c}$ between low-spin atoms. Phase transitions occur for $\beta \omega_{c}>2$, which would require quite large values of $\omega_{c} \quad 0.31 \ldots 0.52 \mathrm{eV}$ for temperatures in Earth's lower mantle. 


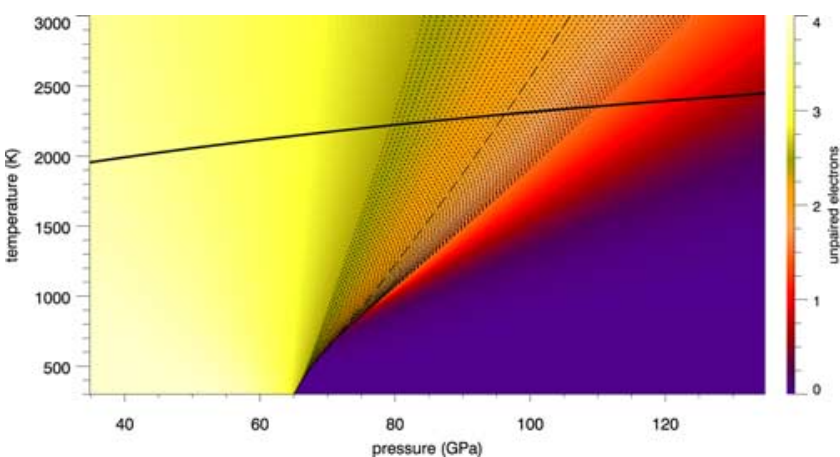

Figure 3. The average number of unpaired electrons for $\mathrm{Mg}_{0.83} \mathrm{Fe}_{0.17} \mathrm{O}$ in the pressure-temperature sector. The dotted contours show the region between 1.5 and 2.5 unpaired electrons. This region clearly widens with increasing temperature. In particular, the iron spin state varies smoothly along a lower mantle geotherm [Brown and Shankland, 1981] given by the solid line. Parameters used for this calculation are given in the text.

where $g_{i}$ is the degeneracy. In the spirit of the mean-field approach, the interaction energy is spatially averaged and the internal energy of the spin ensemble is approximated by

$$
U=-N \sum_{i j} J_{i j} \eta_{i} \eta_{j}+N \sum_{i} E_{i} \eta_{i}
$$

where $J_{i j}$ represents the coupling between an iron atom in spin state $i$ and a nearest neighbor shell of iron atoms in the spin state $j$. The second term incorporates the crystalfield splitting, where $E_{i}$ are the energy levels given in Figure 1. The equilibrium occupation of the spin states is determined from the requirement that the Helmholtz free energy $F=U-T S$ is stationary (minimal) with respect to the probabilities $\eta_{i}$. For a total number of $s$ spin states, this is equivalent to a set of $s$ equations $\partial F / \partial \eta_{i}=0$ that are coupled by the normalization condition $\sum_{i} \eta_{i}=1$, and therefore $s-1$ independent equations are obtained. With the assumption $J_{i j}=J_{j i}$ we write

$$
\beta\left(E_{i}-E_{1}\right)=2 \beta \sum_{j}\left(J_{i j}-J_{1 j}\right) \eta_{j}-\ln \left(\frac{g_{1} \eta_{i}}{g_{i} \eta_{1}}\right) .
$$

[6] With decreasing iron concentration $J_{i j} \rightarrow 0$, and we obtain the solution $\eta_{i}=g_{i} \exp \left(-\beta E_{i}\right) / Z$ with $Z=\sum_{j} g_{j} \exp$ $\left(-\beta E_{j}\right)$, the spin-equilibrium with a continuous crossover between the different spin states.

[7] Depending on parameters like $\beta \omega$ and $\beta J_{i j}$ the free energy can become minimal for many solution vectors. The deepest minimum determines the physically meaningful solution, and the associated probabilities $\eta_{i}$ can change suddenly, e.g., as a function of pressure. Therefore mathematically sharp spin transitions may occur. This behavior will be studied on an ensemble of $\mathrm{Fe}$ (II) atoms with an octahedrally symmetric environment. The crystal-field splitting is caused by the interaction of a given Fe atom with the surrounding atoms including other $\mathrm{Fe}$ atoms. Low-spin $\mathrm{Fe}(\mathrm{II})$ shows a smaller $\mathrm{Fe}-\mathrm{O}$ distance $R$ than high-spin $\mathrm{Fe}(\mathrm{II})$ corresponding to a larger value for the crystal-field splitting. If we associate this energy difference with the coupling energy between low-spin iron atoms in the lattice we may write $\omega_{c}=a(x) \Delta\left(1-R_{L}^{5} / R_{H}^{5}\right)$, where $\Delta \propto R^{-5}$ is the crystal-field splitting in the high-spin state and $a(x)$ depends on the iron concentration $x$. The term in parenthesis is of order unity for reasonable values of $R_{L} / R_{H} \approx 0.9 \ldots 0.95$ [Burns, 1993]. The same $R^{-5}$ dependence leads to $a(x)=$ $x^{5 / 3}$, and we arrive at the estimate $\omega_{c} \approx x^{5 / 3} \Delta$.

[8] The effect of cooperative interactions was modelled by $J_{11}=\omega_{c}$, and all other coupling parameters set to zero. A numerical solution of this model represented by the average number of unpaired electrons calculated from the $\eta_{i}$ values is shown in Figure 2. An increase in the coupling causes a decrease in the crossover region and leads to a mathematically sharp transition for $\beta \omega_{c}>2$. With parameter pairs $(\Delta, T)$ typical for Earth's lower mantle we obtain for the iron concentrations $(1 \mathrm{eV}, 1800 \mathrm{~K}): 0.33,(1.5 \mathrm{eV}$, $1800 \mathrm{~K})$ : 0.26, $(1 \mathrm{eV}, 3000 \mathrm{~K}): 0.44$, $(1.5 \mathrm{eV}, 3000 \mathrm{~K})$ : 0.35 . For the much lower Fe concentrations in lower mantle minerals, sharp transitions between spin states seem therefore unlikely.

\section{Geophysical Implications: Spin Crossover in Ferropericlase}

[9] Ferropericlase, $\mathrm{Mg}_{1-x} \mathrm{Fe}_{x} \mathrm{O}$, is a suitable mineral for a demonstration of the implications of the arguments raised in this paper. The ferrous iron atoms occupy a crystallographic site with octahedral symmetry. We will use a third-order Birch-Murnaghan equation of state (EOS) with $K_{0 T}=\left(K_{0 S}+\right.$ $\left.T\left(\partial K_{S} / \partial T\right)\right) /(1+\alpha \gamma T), K_{0 S}=166 \mathrm{GPa}$, and $\partial K_{S} / \partial P=4.2$ [Kung et al., 2002] (values for $x=0.17$ ). The effect of temperature will be modeled by $\partial K_{S} / \partial T=-0.019 \mathrm{GPa} / \mathrm{K}$ [Sinogeikin et al., 2000], the Grüneisen parameter $\gamma=1.35$ for the investigated range $0.67 \leq V / V_{0} \leq 0.89$ [Speziale et al., 2001], and the thermal expansion coefficient $\alpha=$ $\left(2.763 \cdot 10^{-5} \mathrm{~K}^{-1}+1.439 \cdot 10^{-8} \mathrm{~T} \cdot \mathrm{K}^{-2}\right.$ ) [Fiquet et al., 1999] (values for $\mathrm{MgO})$. The results of $\left(\mathrm{FeMg}_{12} \mathrm{O}_{14}\right)^{2-}$ cluster calculations [Sherman, 1991] indicate that the volume dependence of the crystal-field splitting is given by $\Delta \propto V^{-\xi}$ with $\xi=1.5$, and therefore $\omega=\Delta(V)-\Lambda=$ $\Delta_{0}\left\{\left(V_{0} / V\right)^{\xi}-\left(V_{0} / \bar{V}\right)^{\xi}\right\}$, where $\bar{V}$ is the volume at $65 \mathrm{GPa}$ and $300 \mathrm{~K}$, the reported spin transition in ferropericlase

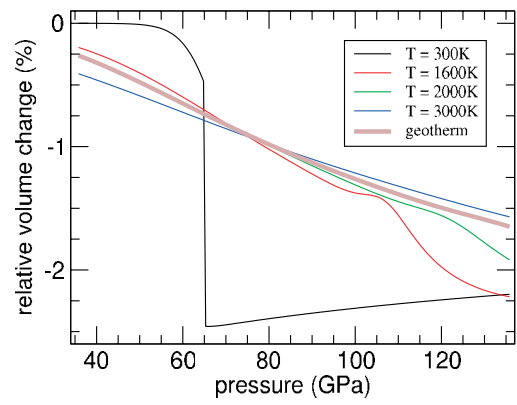

Figure 4. The relative volume change for $\mathrm{Mg}_{0.83} \mathrm{Fe}_{0.17} \mathrm{O}$ with increasing pressure for several isotherms and a lower mantle geotherm [Brown and Shankland, 1981]. The volume change was calculated under the assumption that the equation of state does not change across the spin crossover. Parameters used for this calculation are given in the text. 
[Badro et al., 2003] (values for $x=0.17$ ). The volume and the crystal-field splitting at ambient conditions are $V_{0}$ and $\Delta_{0}=1.35 \mathrm{eV}$, respectively [Goto et al., 1980]. The coupling parameter will be estimated by $\omega_{c} \approx x^{\xi} \Delta(V)$ and $\omega_{c 0} \approx$ $0.09 \mathrm{eV}$ for $x=0.17$ and ambient conditions. For the calculations, we used the slightly more conservative value of $\omega_{c 0}=0.1 \mathrm{eV}$.

[10] In Figure 3, we show the results obtained with the presented mean-field theory for the number of unpaired electrons (shown as colored contours) and thus the spin state of $\mathrm{Fe}(\mathrm{II})$ in $\mathrm{Fp}$ in pressure-temperature space. At $300 \mathrm{~K}$, our calculations predict that the change from the high-spin state (4 unpaired electrons, light yellow) to the low-spin state (0 unpaired electrons, blue) is sharp at $65 \mathrm{GPa}$, in agreement with recent $\mathrm{x}$-ray emission spectroscopic measurements [Badro et al., 2003]. However, under realistic lower mantle conditions, temperatures are much higher. Traversing the $65 \mathrm{GPa}$ isobar with increasing temperature, one can see from Figure 3 that the spin transition becomes broader. A plausible lower mantle geotherm [Brown and Shankland, 1981] has been plotted in Figure 3 and reveals that under typical lower mantle temperatures, the number of unpaired electrons decreases over a pressure interval of more than $30 \mathrm{GPa}$ (equivalent to a depth range of $\approx 700 \mathrm{~km}$ ). In other words, there is no abrupt spin transition in Fp under lower mantle pressures and temperatures. The width of the spincrossover region depends on the chosen parameter set. We tested our model by calculating the logarithmic derivatives $\partial \ln w / \partial \ln p$ of the width $w$ with respect to each parameter $p$. The results in the format (parameter:derivative) are $\xi:-1.7, K_{0 S}: 1.39, \partial K_{S} / \partial P: 1.87, \partial K_{S} / \partial T: 0.61, \alpha_{1}: 0.38$, $\alpha_{2}:-0.074, \gamma:-0.32, \Delta_{0}:-1.2, \omega_{c 0}:-0.23$, transition pressure at $300 \mathrm{~K}: 0.8$ and show that the model is robust in predicting a wide spin-crossover region.

[11] We also calculated the volume reduction with respect to the EOS using parameters determined well below the spin-transition pressure of $65 \mathrm{GPa}$. The ensemble of $\mathrm{Fe}$ atoms exerts an internal pressure given by $-\partial F / \partial V$, the volume derivative of the free energy defined by equations (1) and (2). The externally applied pressure corrected by this internal pressure gives rise to a slightly different volume via the EOS chosen for this material. The relative volume change due to this effect is shown in Figure 4. At $300 \mathrm{~K}$, the volume reduction is about $2.3 \%$ and occurs within a narrow pressure range. However, the volume reduction along the geotherm is smaller and is a smoothly varying function with pressure.

[12] The behavior of $\mathrm{PV}$ is more complex, as it may contain a significant fraction of ferric iron under lower mantle conditions [e.g., McCammon, 1997]. Even though the iron may occupy octahedrally and 8-12 coordinated sites, most of the iron likely resides in the $8-12$ coordinated site, which is expected to show the smaller crystal-field splitting energy $\Delta[$ Burns, 1993]. The pressure derivative of the crystal-field splitting, $\mathrm{d} \Delta / \mathrm{d} P=\xi \Delta / K$, is generally smaller for $\mathrm{PV}$ than for $\mathrm{Fp}$ because of its higher bulk modulus $K$ and the smaller $\Delta$. This will lead to a larger pressure range of a spin crossover in $\mathrm{Pv}$ than in Fp even at $300 \mathrm{~K}$. Our model was constructed for highly symmetrical environments. For distorted environments, which might be expected in Pv and possibly also in Fp, the degeneracy of the energy levels of possible spin states is lifted, and the spin crossover region is broadened even more. A gradual change in the spin state of iron in $\mathrm{Pv}$ to pressures above $100 \mathrm{GPa}$ has recently been observed at $300 \mathrm{~K}$ using $\mathrm{x}$-ray emission spectroscopy [ $\mathrm{Li}$ et al., 2004] and synchrotron Mössbauer spectroscopy [Jackson et al., 2005].

[13] Independently of the character of the transition at $300 \mathrm{~K}$, our calculations predict that the high-spin to lowspin crossover regions and the associated volume changes in $\mathrm{Pv}$ and Fp occur over a very broad pressure region at typical lower mantle temperatures. Our results imply that any chemical and physical changes associated with this crossover region, such as iron partitioning and elastic properties [e.g., Kobayashi et al., 2004], would also be gradual. It is also possible that the oxidation state of iron in lower mantle minerals plays an important role in chemical changes associated with this large crossover region of several hundred kilometers depth. Furthermore, the hitherto unknown electronic properties of $\mathrm{Fe}$ in post-silicate perovskite [Murakami et al., 2004] may lead to additional complexities at the base of the lower mantle.

[14] Acknowledgments. This work was supported by the U.S. Department of Energy, Office of Science, Basic Energy Sciences, under Contract No. W-31-109-Eng-38. JMJ acknowledges support from NSF EAR-0135642.

\section{References}

Badro, J., V. V. Struzhkin, J. Shu, R. J. Hemley, H. K. Mao, J. P. Rueff, and C. C. Kao (2003), Iron partitioning in Earth's mantle: Toward a deep lower mantle discontinuity, Science, 300, 789-791.

Badro, J., J. P. Rueff, G. Vanko, G. Monaco, G. Fiquet, and F. Guyot (2004), Electronic transitions in perovskite: possible nonconvecting layers in the lower mantle, Science, 305, 383-386.

Brown, J. M., and T. J. Shankland (1981), Thermodynamic parameters in the Earth as determined from seismic profiles, Geophys. J. R. Astron. Soc., 66, 579-596.

Burns, R. G. (Ed.) (1993), Mineralogical Application of Crystal Field Theory, Cambridge Univ. Press, New York.

Cohen, R. E., I. I. Mazin, and D. G. Isaak (1997), Magnetic collapse in transition metal oxides at high pressure: implications for the Earth, Science, 275, 654-657.

Fiquet, G., P. Richet, and G. Montagnac (1999), High-temperature thermal expansion of lime, periclase, corundum, and spinel, Phys. Chem. Miner., $27,103-111$

Gaffney, E. S., and D. L. Anderson (1973), Effect of low-spin $\mathrm{Fe}^{2+}$ on the composition of the lower mantle, J. Geophys. Res., 78, 7005-7014.

Goto, T., T. J. Ahrens, J. R. Rossman, and Y. Suono (1980), Absorption spectrum of shock-compressed $\mathrm{Fe}^{2+}$-bearing $\mathrm{MgO}$ and the radiative conductivity of the lower mantle, Phys. Earth Planet. Inter., 22, $277-288$

Gütlich, P., and H. A. Goodwin (Eds.) (2004), Spin Crossover in Transition Metal Compounds I-III, Springer, New York.

Jackson, J. M., W. Sturhahn, G. Shen, J. Zhao, M. Y. Hu, D. Errandonea, J. D. Bass, and Y. Fei (2005), A synchrotron Mössbauer spectroscopy study of $(\mathrm{Mg}, \mathrm{Fe}) \mathrm{SiO}_{3}$ perovskite up to $120 \mathrm{GPa}$, Am. Mineral., 90, 199205, doi:10.2138/am.2005.1633

Kellogg, L. H., B. H. Hager, and R. D. van der Hilst (1999), Compositional stratification in the deep mantle, Science, 283, 1881-1884.

Kobayashi, H., T. Kamimura, D. Alfè, W. Sturhahn, J. Zhao, and E. E. Alp (2004), Phonon density of states and compression behavior in iron sulfide under pressure, Phys. Rev. Lett., 93, doi:10.1103/ PhysRevLett.93.195503.

Kung, J., B. Li, D. J. Weidner, J. Zhang, and R. J. Liebermann (2002), Elasticity of $\left(\mathrm{Mg}_{0.83} \mathrm{Fe}_{0.17}\right) \mathrm{O}$ ferropericlase at high pressure: Ultrasonic measurements in conjunction with x-radiation techniques, Earth Planet. Sci. Lett., 203, 557-566.

Li, J., et al. (2004), Electronic spin state of iron in lower mantle perovskite, Proc. Natl. Acad. Sci., 101, 14,027-14,030, doi:10.1073/ pnas.0405804101.

Liu, L.-G. (1975), Post-oxide phases of olivine and pyroxene and mineralogy of the mantle, Nature, 258, 510-512.

McCammon, C. A. (1997), Perovskite as a possible sink for ferric iron in the lower mantle, Nature, 387, 694-696. 
McCammon, C., J. Peyronneau, and J.-P. Poirier (1998), Low ferric iron content of $(\mathrm{Mg}, \mathrm{Fe}) \mathrm{O}$ at high pressures and temperatures, Geophys. Res. Lett., 25, 1589-1592.

Murakami, M., K. Hirose, K. Kawamura, N. Sata, and Y. Onishi (2004), Post-perovskite phase transition in $\mathrm{MgSiO}_{3}$, Science, 304, 855-858.

Shannon, R. D., and C. T. Prewitt (1969), Effective ionic radii in oxides and fluorides, Acta Crystallogr., Sect B Struct. Crystallogr. Cryst. Chem., 25, 925-946.

Sherman, D. M. (1988), High-spin to low-spin transition of iron (II) oxides at high pressures: Possible effects on the physics and chemistry of the lower mantle, in Structural and Magnetic Phase Transitions in Minerals, edited by S. Ghose, J. M. D. Coey, and E. Salje, pp. 113-128, Springer, New York.

Sherman, D. M. (1991), The high-pressure electronic structure of magnesiowustite (Mg, Fe)O: Applications to the physics and chemistry of the lower mantle, J. Geophys. Res., 96, 14,299-14,312.

Sherman, D. M., and H. J. F. Jansen (1995), First-principle prediction of the high-pressure phase transition and electronic structure of $\mathrm{FeO}$ : Implications for the chemistry of the lower mantle and core, Geophys. Res. Lett., 22, 1001-1004.

Sinogeikin, S. V., J. M. Jackson, B. O’Neill, J. Palko, and J. D. Bass (2000), Compact high-temperature cell for Brillouin scattering measurements, Rev. Sci. Instrum., 71, 201-206.
Speziale, S., C.-S. Zha, T. S. Duffy, R. J. Hemley, and H.-K. Mao (2001), Quasi-hydrostatic compression of magnesium oxide to $52 \mathrm{GPa}$ : Implications for the pressure-volume-temperature equation of state, J. Geophys. Res., 106, 515-528.

Trampert, J., F. Deschamps, J. Resovski, and D. Yuen (2004), Probabilistic tomography maps chemical heterogeneities throughout the lower mantle, Science, 306, 853-856.

van der Hilst, R. D., and H. Kárason (1999), Compositional heterogeneity in the bottom 1000 kilometers of Earth's mantle: Toward a hybrid convection model, Science, 283, 1885-1888.

Williams, E. J. (1935), The effect of thermal agitation on atomic arrangement in alloys III, Proc. R. Soc. London, Ser. A, 152, 231-252.

J. M. Jackson, Department of Geology, University of Illinois at UrbanaChampaign, 1301 Green Street, Urbana, IL 60801, USA.

J.-F. Lin, Geophysical Laboratory, Carnegie Institution of Washington, 5251 Broad Branch Road NW, Washington, DC 20015, USA.

W. Sturhahn, Advanced Photon Source, Argonne National Laboratory, 9700 South Cass Ave, Lemont, IL 60439, USA. (sturhahn@aps.anl.gov) 